\title{
Correction de copies : compte-rendu de stage et applications pédagogiques
}

\section{Brigitte Pons}

\section{(2) OpenEdition}

\section{Journals}

Édition électronique

URL : http://journals.openedition.org/edc/3253

DOI : $10.4000 /$ edc.3253

ISSN : 2101-0366

Éditeur

Université de Lille

Édition imprimée

Date de publication : 1 février 1986

Pagination : 137-151

ISSN : $1270-6841$

Référence électronique

Brigitte Pons, «Correction de copies : compte-rendu de stage et applications pédagogiques », Études de communication [En ligne], 7 | 1986, mis en ligne le 01 avril 2012, consulté le 21 décembre 2020 URL : http://journals.openedition.org/edc/3253 ; DOI : https://doi.org/10.4000/edc.3253

Ce document a été généré automatiquement le 21 décembre 2020.

(c) Tous droits réservés 


\title{
Correction de copies : compte-rendu de stage et applications pédagogiques
}

\author{
Brigitte Pons
}

1 L'article qui suit est volontairement divisé en deux parties bien distinctes.

2 Je l'ai rédigé à la demande des animateurs du stage, avec comme projet de départ un article unique, compte-rendu d'une tentative pédagogique qui tenait compte des réflexions suscitées par le stage lui-même.

3 En écrivant, il m'est très vite apparu difficile sinon impossible pour moi de mêler de façon satisfaisante le travail et la réflexion effectués au cours du stage et la démarche pédagogique que j'avais adoptée auprès de mes élèves.

4 Entre ces deux lieux, celui du stage et la classe, les interactions étaient nombreuses. Mais ma position était bien différente. D'un côté, j'étais l'"apprenant", celui qui vient pour savoir et qui se trouve là parce qu'il s'interroge. D'un autre côté, je suis l'"enseignante", celle qui transmet un savoir, suscite et constate une réflexion. Entre ces deux places, il y a aussi celle du travail chez soi, de la réflexion lente et longue, de la mise en place d'une progression, il y a aussi la "mémoire de papier" de mon travail que j'enseigne.

5 Il m'est apparu difficile de décrire une tentative faite en classe en la ponctuant, de ci de là, de retours en arrière expliquant ce qui revenait au stage, ou à ma réflexion, ou à l'expérience des années antérieures.

6 C'est pour cela que j'ai scindé mon travail en deux parties. Il m'apparaissait plus productif d'offrir au lecteur, d'un côté, un aperçu de ce que le stage recouvrait, d'un autre côté une possibilité d'application pédagogique.

\section{Première partie}

7 Ce stage inscrit au P.A.F. pour l'année scolaire 1985-1986 s'est déroulé en trois périodes de deux jours chacune, en novembre et décembre 1984 et en avril 1985. 
8 Il a permis dans un premier temps à tous les participants de mettre en commun ce moment important quantitativement et solitaire de la vie d'un prof: la correction de copies. Le constat n'était pas gai : un ras-le-bol généralisé, la conscience du temps perdu à corriger, beaucoup de doutes quant à l'efficacité de nos corrections auprès de nos élèves. En résumé, le sentiment, quelque part, que la correction de copies, "ça ne vaut pas le coup",

9 Si ce moment pouvait revêtir un aspect gratifiant par la reconnaissance commune d'attitudes individuelles, il nous a fallu tout de même aller plus loin. Se souvenir de nos réactions d'élèves face aux copies corrigées que nous rendaient nos professeurs, faire le bilan des réactions connues de nos élèves face à nos propres corrections. Cette double réflexion allait dans le même sens que notre lassitude de prof-correcteur de copies.

10 Ces trois points de vue peu encourageants, nous y étions déjà sensibilisés en nous inscrivant à ce stage mais, là, nous pouvions aborder le problème en commun, dans toute son ampleur.

11 Il nous fallait aller plus loin que l'observation globale des réactions.

12 Bernard Delforce nous a proposé des copies d'élèves à corriger. L'enthousiasme était loin d'être général, mais la démarche efficace. Le fait de corriger et de s'observer en train de corriger, la mise en commun des corrections individuelles, tout cela a permis de préciser, de mettre au jour nos pratiques individuelles et ce qu'elles avaient en commun: normativité, corrections en grande proportion négatives ("je note ce qui ne convient pas, plutôt que ce qui convient"), désir d'exhaustivité dont nous avons pu mesurer l'inefficacité, entre autres constatations.

13 L'exercice suivant qui nous a été proposé consistait à reprendre une copie d'élève et à proposer plusieurs possibilités d'amélioration de cette copie. Nous avions toujours pour consigne, ici comme dans l'exercice précédent, de nous observer en train de faire ce travail. Très vite, la contrainte de cette consigne a débouché sur un intérêt neuf face aux démarches d'apprentissage, aussi bien les nôtres que celles de nos élèves.

Et c'est bien là que m'est apparu, en tant que stagiaire, le premier intérêt de ce stage, intérêt en situation, mais aisément transférable dans la vie de tous les jours d'un prof. Depuis longtemps déjà, j'étais affrontée, comme tous mes collègues, au problème qui consiste à "faire passer" le plus efficacement possible un cours auprès de mes élèves. Mais cette réflexion, par ses manques, n'était pas inscrite à l'intérieur d'une progression globale. Elle se faisait au coup par coup, variant avec le type d'exercices proposés en classe, avec le niveau des élèves, s'améliorant au fur et à mesure de l'expérience, sans pour autant acquérir une réelle cohérence, aisément perceptible pour moi. L'impression de "bricolage" qui s'en dégageait me laissait souvent insatisfaite.

15 Mettre très fortement l'accent sur une observation des démarches d'apprentissage, les miennes, mais aussi celles des élèves, sur une interrogation vis à vis de ces démarches, tenter de les éclaircir par une perception plus fine pour pouvoir mieux les comprendre, c'est déjà se donner les moyens de construire une démarche globale cohérente par rapport à un apprentissage donné.

16 Avec les leçons de l'expérience, c'est également comprendre que quand démarche il y a, elle ne peut pas pour autant être figée, établie une fois pour toutes, répétée d'une année sur l'autre pour tous les élèves et tous les professeurs. Les élèves ne sont pas tous les mêmes, aussi bien pris individuellement que dans le groupe-classe, les professeurs ne 
sont pas tous les mêmes et chacun accomplit son travail à l'aide de ses capacités/ spécificités individuelles.

17 Cette prise de conscience était fort déroutante. Il n'y a pas là don de "recettes de cuisine" qui, si efficaces soient-elles, n'en restent que ponctuelles. Il s'agit ici de se pencher sur un en deçà du contenu même du cours, sur la façon dont les uns s'approprient le savoir et dont les autres le transmettent. Bref, le problème de la correction des copies nous a amené à déboucher sur une question pédagogique bien plus générale, un travail qui est tout le temps à faire, qui nous oblige à nous intéresser de très près (à l'aide de peu d'outils sinon l'observation) à la culture des élèves, à la nôtre, aux relations que les uns et les autres entretiennent avec le savoir, à nos fonctionnements qui nous paraissent évidents et dont nous voyons qu'ils ne le sont pas quand le message ne passe pas, mais qui ne sont pas pour autant clairs quand le message passe.

18 Cette réflexion peut, tôt ou tard, prendre l'aspect d'une remise en cause personnelle. Elle a le risque de devenir quelque peu déstructurante. Elle présente toutefois l'avantage de ne pas jeter totalement à bas ce que nous faisons depuis des années pour y substituer, en mêmes lieu et place, une série de propositions catapultées par quelques personnes dont nous nous accordons pour penser "qu'elles sont un peu plus calées que nous". Ce risque peut être évité puisqu'on ne nous présente rien comme étant la panacée. L'analyse porte toujours là sur notre propre démarche et celle de nos élèves.

19 Si cela dérange trop, il est possible de s'en tenir à ses pratiques habituelles. Les stagiaires présents à la deuxième session (et ils étaient toujours très nombreux) ont pu partager leur déroute, aussi bien face à leur enseignement que face à la correction des copies envisagée d'un œil neuf.

20 Les exercices individuels et collectifs se sont succédés, qui quelque part nous sécurisaient à nous obliger à répondre à des consignes précises et limitées, mais nous replongeaient aussi dans les affres d'interrogation à l'analyse. Toutefois, dès cette deuxième session, nous avons pu voir avec plaisir qu'il y avait, dans nos pratiques et notre réflexion, de quoi glaner de droite et de gauche pour aboutir à la construction d'une démarche plus globale et plus cohérente. La mise en commun du travail plus ou moins secret, si ce n'est honteux, qu'est la correction de copies, la prise de conscience de l'importance des consignes de travail données aux élèves et la réflexion commune qui a suivi nous ont permis de clarifier ensemble l'idée d'une démarche globale. Chacun individuellement a pu ensuite juger de quelle façon il pouvait, par rapport à ce qu'il faisait ou se sentait capable de faire, améliorer sa propre démarche pédagogique. Nous avons pu en parler avec les uns et les autres, entendre les expérimentations, faites par certains, aussi tenter de nous les approprier, hors du stage, par nos propres expérimentations.

21 Les "pistes" proposées étaient multiples : réflexion sur les démarches d'apprentissage et sur l'importance des consignes données aux élèves, je l'ai déjà dit. Mais aussi observation/ réflexion sur le mode de fonctionnement de la lecture, observation/réflexion sur les approches thématiques avec ce qu'elles offrent de possibilités et de restrictions, observation/réflexion sur la façon dont "on" fait le plan d'un texte, ce qui nous a amené à remettre très fortement en cause la voix unique du professeur (nous étions nombreux et il y avait presque autant de plans proposés que de stagiaires). Nous avons aussi travaillé et réfléchi ensemble sur ce qui concerne nos pratiques face aux deux exercices précis que sont le résumé et l'exercice de dissertation/essai/discussion. Cela nous a permis de trouver dans nos propres consignes et dans les modèles que nous proposons aux élèves les germes des "tristes" travaux qu'ils nous rendent. Ensemble nous avons cherché des 
solutions efficaces à ce que nous qualifions d"'aporie" dans les copies d'élèves, en réfléchissant aux consignes données, au traitement que nous-mêmes faisons subir aux textes, à nos propres représentations de ce qu'est un "bon" devoir, à celles des élèves et à la façon dont ils estiment devoir s'y prendre pour séduire le correcteur en vue d'une bonne note, aux préjugés importants qui entachent l'idée qu'ils se font sur la façon dont les copies sont notées (et ils ne sont pas tous infondés), même si nous avions accepté les propos de Bernard Delforce sur "la réalité incontournable de l'examen".

Cela nous a amené à nous méfier de l'efficacité toute relative des démarches en pour/ contre, avantages/inconvénients. Nous avons lu et relu de nombreux textes, textes d'élèves en vue d'un travail de réécriture, dont certains avaient pu mesurer l'efficacité par l'expérimentation ; extraits de journaux, sujets d'examen également, dans le but d'en discerner le fonctionnement. La proposition faite alors d'un travail sur les modèles textuels a été bien accueillie, même si nous sommes restés quelque peu frustrés par le peu de temps imparti à cette tâche dans le cadre du stage.

Toujours est-il que nous avons quitté le stage nantis d'outils de réflexion, d'un important matériel de travail, ayant acquis quelque part un regard neuf sur la pédagogie. L'intitulé du stage, volontairement limité, prêtait alors pour nous à sourire.

Mais le plus important restait à expérimenter, car c'est sur le terrain même de l'établissement scolaire que le travail pouvait porter ses fruits.

\section{Deuxième partie}

La réflexion tous azimuts produite par le stage avait bien besoin d'être clarifiée et surtout de déboucher sur une mise en pratique cohérente, autant que faire se peut.

Si, personnellement, je désirais consacrer plus de temps et d'attention que d'ordinaire à l'observation des élèves au travail ainsi qu'à mes propres démarches, j'avais aussi le désir de pouvoir utiliser rapidement certains matériaux anciens revus et corrigés après réflexion et des matériaux neufs acquis durant le stage.

Cette démarche apparaissait difficile dans la mesure où l'année était déjà bien entamée, où la progression de travail avec les élèves était déjà mise au point depuis longtemps. Toutefois, j'ai au moins pu faire quelques tentatives d'exercices limités avec des classes du second cycle, et une expérimentation plus globale avec des étudiants de formation continue auprès desquels j'assurais des cours de janvier à avril... C'est bien sûr seulement à partir de septembre 1985, en second cycle, que la démarche a pu commencer à être plus globale et cohérente, inscrite dès le départ dans une large progression. J'en jette ici les grandes lignes, non pas en la présentant comme un modèle, mais comme une des adaptations possibles qui a pu exister par les acquis du stage, à partir de mes réflexions et de mes spécificités.

C'est sur l'apprentissage de l'écrit qu'a porté mon effort, surtout en classe de seconde, au début même du travail sur les textes non narratifs.

Le point de départ vital du travail me semble être depuis longtemps le problème de la lecture.

Face à un type de textes (textes non narratifs) dont les élèves ont peu coutume au C.E.S., on a souvent affaire à l'effarement. L'ampleur du texte, les difficultés de vocabulaire, voire les difficultés syntaxiques, effrayent. L'accent est tout de suite mis sur le sens, qui 
leur parait complexe, quand il apparaît. Ils ont souvent l'idée que tout cela n'est que verbiage et que de toute évidence ils ne parviendront pas à en faire autant.

Ma volonté a donc d'abord été de faire une progression dans les difficultés à aborder, de les amener à réfléchir à la façon dont on lit un texte, et à la façon dont il est "fabriqué".

Pour cela, il fallait d'abord les rendre conscients du fonctionnement de la lecture, de son mécanisme.

33 Je leur ai donné tout d'abord un texte long, dense, à lire en temps limité en leur demandant d'aller vite, de ne pas laisser l'œil revenir en arrière. Le bilan fait ensemble a dégagé une grande perte de compréhension et la nécessité, pour tout lecteur, de ces retours en arrière de l'œil qui sont si utiles à la compréhension. Seul le thème général du texte était dégagé, ou bien un moment particulier, qui avait retenu l'attention de tel ou tel élève en évoquant un point qui avait une résonance pour lui.

L'exercice suivant adoptait une démarche inverse. Un texte long et dense, à lire en temps illimité, chacun à son rythme, le professeur servant occasionnellement de dictionnaire. Au bilan, le tableau était couvert d'idées particulières toutes présentes dans le texte, mais les élèves ont pris conscience de leur incapacité à verbaliser la démarche générale de l'auteur du texte.

Ces deux exercices ont permis de concrétiser le fonctionnement de la lecture et de déculpabiliser tous ceux qui pensaient qu'il n'y avait qu'eux qui ne comprenaient pas "tout" en lisant un texte. Mais il fallait avancer et appréhender quelques façons efficaces de lire.

J'ai alors proposé aux élèves un long travail qui permettait d'aboutir à l'établissement d'un modèle textuel.

Ils ont eu en mains un texte dont certaines parties avaient disparu. Ils l'ont lu et relu tel que, puis ont pris en notes, paragraphe par paragraphe, ce qui était imprimé. Ils ont vite réalisé qu'il s'agissait des points de départ et d'arrivée des idées du texte. Ils ont analysé le fait que certains paragraphes étaient présentés de façon complète, sans "blancs". Ils se sont expliqués pourquoi. Ils ont enfin supposé que ce qui était "gommé" correspondait à l'illustration, à ce qu'ils appellent le remplissage.

39 Je leur ai alors fourni le texte exhaustif et ils ont pu vérifier que leur hypothèse était fondée. Ils ont alors pris note (avec une encre de couleur différente, c'était plus facile pour l'efficacité du travail) des "blancs" du texte de départ.

Les deux travaux se complétant, ils ont abouti au texte lui-même et ont pu réaliser son fonctionnement. D'un côté les grandes articulations du discours, de l'autre les illustrations, les preuves et les redites.

41 Un large temps a été consacré au bilan de ce gros travail. Ils se sont alors aperçu que le texte prenait difficilement sens à ne pas être traité de façon complète tout en reconnaissant que le fait de scinder en deux le travail les avait aidé à scinder les difficultés et à ne pas être écrasés par le volume même du texte. Ils ont appris à reconnaître l'utilité de mêler articulations du discours et "remplissage". Ils ont trouvé le point de départ du texte et ont reconnu les diverses façons dont l'auteur usait pour avancer dans sa démarche de pensée et pondérer son discours. Ce qui les a le plus surpris, c'étaient les redites, qui ne leur étaient pas du tout apparues à première lecture, qu'ils ont jugées très sévèrement tout d'abord ("il ne sait pas écrire, il se répète"), puis qu'ils ont 
estimé nécessaires pour la bonne compréhension du texte. Ils ont également trouvé qu'il y avait peu d'idées dans ce texte et cela les a d'autant plus surpris qu'il était volumineux.

Je leur ai alors proposé de lire deux textes de plus faible ampleur et d'en recenser la progression, comme ils l'avaient fait pour le premier. Ils l'ont fait et se sont aperçus qu'ils arrivaient aux mêmes constatations. Une progression de pensée qui part d'un constat illustré pour passer à une explication plus ou moins exhaustive qui permet à l'auteur d'aboutir à des propositions. Pour eux, l'établissement d'un modèle de texte, ce qui me paraissait primordial. Ils avaient appris à reconnaître un texte, et du même coup, se sentaient moins démunis face aux nouveaux textes proposés. En outre, si le sens leur semblait toujours fort important, ils étaient sensibilisés à la forme qui pouvait les rassurer avant toute démarche de travail.

Afin de bien "asseoir" les acquis de ce travail, je leur ai proposé un autre type d'exercice.

A plusieurs reprises, ils ont eu à manier des polycopiés où figuraient un certain nombre de phrases (de 8 à 24) qui étaient des schémas de textes (dissertations) produits par d'autres élèves. Chaque item était numéroté et n'apparaissait pas à sa place exacte. Il s'est agi pour eux d'apprendre à opérer un classement, de reconnaître un sens, à travers les mots-outils utilisés, les temps employés, en mettant en relation certains termes, en réfléchissant à l'utilisation des démonstratifs, par exemple.

Ce travail fait, ils l'ont recommencé sur des textes de baccalauréat cette fois, ceux proposés dans le cadre du sujet $\mathrm{n}^{\circ} 1$. J'avais "désordonné" des paragraphes entiers. Les difficultés augmentaient à chaque exercice dans la mesure où j'introduisais peu à peu des textes qui s'éloignaient de façon de plus en plus sensible du modèle qu'ils avaient appris à reconnaître, sans toutefois être fondamentalement différents. Il semblerait en effet (je ne peux en juger encore, n'ayant étudié qu'une quinzaine de textes) que le fonctionnement, le modèle textuel décrit plus haut puisse recouvrir la majeure partie des textes proposés aux élèves.

Les deux grands axes de ce travail, à savoir reconnaître un modèle textuel et apprendre à le reconstruire s'apparentent à mon avis à la démarche d'apprentissage de certaines techniques. En mécanique, en dessin industriel, matières dont je peux parler avec mes collègues puisque j'enseigne en lycée technique, ils ont à observer, puis à décrire des pièces précises, à les "démonter" visuellement et sur papier avant de pouvoir à leur tour produire des pièces similaires qui ne varient au départ que par leurs dimensions, la conception globale des pièces se retrouvant d'une étude à l'autre.

Il me semble qu'on retrouve là aussi la démarche générale du cours de physique, jusqu'au niveau de la première (pour ce que j'en sais) et de ce qu'on appelait il y a quelques temps encore la technologie.

48 Ce "jeu de construction" est reconnu par les élèves, qui parviennent vite à s'apercevoir que s'il est utilisé en cours de français, il est aussi utilisé ailleurs. Ils tirent de cette constatation une certaine confiance dans la mesure où ils manifestaient auparavant une désorientation face à des matières différentes qui paraissaient correspondre chacune à des lois et à des fonctionnements très différents.

Je tire un autre enseignement du travail de construction d'un texte désordonné, qui me paraît non négligeable.

L'angoisse habituelle d'un élève moyen de seconde réside dans le fait de savoir si "on va continuer à faire des dictées" et si "la grammaire ça compte". 
51 En apprenant à ré-ordonner des textes, en se fixant sur cette consigne, en "jouant" (car c'est presque toujours comme cela qu'ils prennent cet exercice) à retrouver un ordre logique, qui n'est pas forcément toujours unique, mais qu'ils ont en tous cas à justifier, à défendre, face à leurs camarades et face au professeur, ils utilisent par là même, sans que cela semble les gêner, sans que je les y engage, la syntaxe, l'orthographe, la ponctuation et la présentation du texte. (Je reviendrai plus tard sur ces deux derniers points).

Ils en arrivent en un mois et demi de temps à une situation paradoxale : effrayés par la grammaire, ayant décidé de ne plus en parler, ils s'en servent et complètent leurs manques en ayant recours à leurs manuels de grammaire ou en me posant des questions strictement grammaticales, ce qui leur fait gagner du temps, à leurs yeux, sur la fastidieuse recherche dans le manuel.

Bref, ils prennent conscience de l'utilité de la grammaire, de l'efficacité qu'on a à la connaître, et ceci transparaît ici, mais aussi bien quand nous abordons les textes littéraires qui sont au programme des classes cette année. Ils n'ont plus peur de l'outilgrammaire. Ils s'en servent en se l'appropriant.

J'ai évoqué tout à l'heure la question de la ponctuation et de la présentation du texte, il me faut maintenant y revenir. En réordonnant des textes beaucoup plus qu'en en lisant, les élèves prennent conscience que ces deux points sont importants et le disent.

Il y a très vite de leur part une demande d'information technique sur la ponctuation. Le professeur s'exécute et une fiche de ponctuation est établie. Ils peuvent alors affronter l'exercice casse-tête qui consiste à mettre la ponctuation sur un devoir d'élève où elle a été intégralement ôtée. C'est un exercice long et fastidieux. C'est pénible pour eux au départ : les dix premières lignes, après lecture globale, sont lentes à faire, mais très vite, ils accélèrent le rythme pour terminer avec une vélocité peu commune qui n'entrave en rien la justesse des remarques qu'ils font de vive voix.

Cet exercice reste extrêmement efficace, je m'en aperçois vite à travers leurs propres travaux d'écriture et surtout, à l'évidence, en les écoutant se reprocher mutuellement des "absences" ou "erreurs" de ponctuation quand ils ont à lire le texte d'un des camarades avec qui ils travaillent.

57 En ce qui concerne la présentation du texte, il semble qu'il faille d'abord les amener à remettre en cause l'idée bien ancrée qu'ils ont en tête qu"'il y a u n e idée par paragraphe et un paragraphe par idée". Pour ce faire, il faut en passer par l'observation, sous un autre angle, des textes de bac qu'ils ont déjà travaillés.

58 Ils relisent le premier texte travaillé en recherchant le nombre d'idées et en constatant vite qu'il ne "colle" pas avec leur thèse. Le constat est rapide : ce texte est un "mauvais" texte, "mal écrit". A faire ce travail sur plusieurs textes différents, ils s'aperçoivent alors enfin que leur thèse de départ n'est pas tout à fait juste, que l'écriture n'est pas si mécaniste et ils ne se privent pas de dire qu'on leur demande de réaliser un exercice difficile que peu d'auteurs parviennent à accomplir. Ils classent les textes étudiés (5, 6 suffisent) dans l'ordre, depuis celui qui s'approche le plus de la règle "un paragraphe par idée, une idée par paragraphe", jusqu'à celui qui en est le plus éloigné. Ils concluent que la règle n'est pas stricte, que les textes tendent vers cette règle et que la plus grande lisibilité est afférente au texte qui la respecte sensiblement. Ils s'expliquent les distorsions quant à cette règle par la presque nécessaire concaténation de paragraphes de taille sensiblement identiques. Ces deux derniers constats leur servent d'enseignement pour leur propre travail d'écriture, de plus en plus présent à leur esprit au fur et à mesure 
des exercices. En rendant leurs devoirs, ils m'expliquent ou même ils inscrivent sur une annexe jointe (on comprendra ensuite pourquoi) dans quelle mesure ils ont essayé de mettre une idée par paragraphe et un paragraphe par idée, mais aussi pourquoi, ponctuellement, ils ne l'ont pas fait. Ce qui ressort surtout de ces remarques, c'est qu'ils ont saisi un élément essentiel du travail d'écriture, dans la mesure où ils mettent l'accent sur les critères de lisibilité.

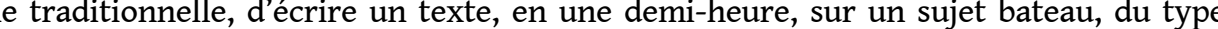
pour/contre. La brièveté de la consigne et les réponses que j'avais pu apporter à leurs, questions ne semblaient guère les avoir mis en confiance. Ils n'ont pas compris pourquoi je disais que nous nous resservirions de cet exercice plus tard et je n'ai pas estimé le moment propice à l'explication complète de la démarche pédagogique adoptée. Ils n'ont pas davantage compris pourquoi l'exercice ne serait pas noté, pourquoi sa "taille" m'importait peu, comment il fallait le faire. Mes "comme tu veux" ne les ont pas rassurés, loin de là, et encore moins les "je sais" que je leur rendais quand ils me manifestaient leur incapacité à faire l'exercice. Durant les quinze jours qui ont suivi, j'ai fait l'objet de multiples questionnements quant aux "devoirs" faits en début d'année. J’ai pu, petit à petit, au fur et à mesure où nous avancions dans le travail sur le modèle textuel, leur apporter des réponses. précédentes, ils ont tout de même été très surpris: pas de note, leurs fautes d'orthographe corrigées, simplement des chiffres de-ci delà qui renvoyaient à une feuille annexe où soit je leur posais des questions sur un point précis de leur raisonnement, soit j'énonçais ce que j'avais cru comprendre et leur demandais de me dire si c'était bien cela qu'ils avaient voulu exprimer. souvent à se souvenir de ce qu'ils avaient voulu dire. Ils ont vite compris que, plus que la mémoire du passé, c'était bien le fait de recommencer leur démarche de pensée qui allait les aider à comprendre leurs travaux, à répondre aux questions que je leur avais posées par écrit.

66 Le temps de familiarisation avec l'exercice passé, ils ont tenté rapidement d'améliorer leurs textes, ils ont trouvé de nouvelles voies d'expression, et je leur ai alors proposé de ré-écrire ce travail, ce qu'ils ont fait. Chacun a mesuré, par lui-même, l'écart existant entre les deux textes, et c'est cet écart qui a pour moi été sanctionné par une note, en corrigeant la ré-écriture du travail, en regard de la "première mouture".

67 L'idée de noter l'écart d'un travail à un autre a été l'une des plus difficile à faire comprendre aux élèves. C'est un type de correction, de "sanction" encore plus (j'entends 
la sanction-note) à laquelle ils ne sont pas habitués. Je l'avais déjà fait auparavant dans un exercice de ré-écriture de remise en ordre d'un texte, et à l'occasion d'un résumé. C'est un élève, acquis à cette notation, qui l'a finalement bien mieux explicitée que moi auprès de ses camarades. Il a parlé des courses de chevaux et des handicaps de poids donnés au départ aux meilleurs, justifiant ainsi un système de notation par bonification entre la première mouture et la seconde. Le seul point noir verbalisé par le délégué de classe restait l'incidence de la note des deux moutures sur la moyenne de l'élève. En toute logique, le délégué n'acceptait pas l'idée qu'un "bon" élève, dont la première mouture était correcte "gagne" moins de points à la deuxième mouture (et pour cause, il avait moins de progrès à faire, donc droit à moins de points de bonification) qu'un "mauvais" élève qui lui gagnait gros en s'étant nettement amélioré à la deuxième mouture. Ils ont fait, à ma demande, des moyennes comparatives, et se sont aperçus que le système habituel de notation gardait ses droits puisque les "bons" élèves obtenaient malgré tout de meilleures moyennes même si les écarts de notes, importants à la première mouture, s'étaient considérablement réduits (vers le haut) à la seconde. Rassurés, ils ont pu alors me réconforter en me disant que ce système apparaissait juste tout en étant gratifiant pour les "faibles" qui pouvaient enfin mesurer leurs progrès.

Nous avons eu une longue discussion sur ce point, qui peut paraitre futile à certains, mais qui, en fait, est primordial, au vu du déchaînement de passion qu'il entraîne. La note est pour eux un salaire, la sanction du système dans lequel ils vivent et il me paraît important qu'ils en demandent justification. Ne pas la leur donner, ne pas répondre à leurs questionnements, c'est ne pas tenir compte de l'importance qu'ils accordent à la note, c'est, à terme, justifier la note-loterie et, par voie de conséquence, la minimiser, ainsi que les annotations de correction qui vont avec. C'est donc jouer le jeu de l'inefficacité, cautionner le fait que "la correction, ça ne sert à rien", pour les élèves comme pour le prof.

Quasiment tout le travail de l'année est écrit puis ré-écrit, noté et annoté, puis renoté et ré-annoté, en ce qui concerne au moins la dissertation/essai/discussion.

Je veux maintenant parler ici du travail spécifique du prof. Je sais, j'entends d'ici les objections, je les ai prononcées moi-même: c'est long, très long, fastidieux, très fastidieux, déroutant. Mais, et ce mais est de taille: c'est un travail d'une étonnante rentabilité.

$71 \mathrm{Au}$ départ, il prend énormément de temps. La feuille annexe est une contrainte inhabituelle, un gros maniement de papier, obligatoire pour les exercices de grande taille, pas obligé pour le résumé. La correction elle-même semble partir dans tous les sens, d'autant que ce type de travail tend à exclure la normativité. A quoi servirait ce travail, s'il était normatif?

Très vite, heureusement (j'entends à la quatrième ou à la cinquième pile de copies corrigées ainsi), des automatismes se créent, des formules reviennent. Corriger ainsi, cela s'apprend aussi. Ici encore, il est question de démarches d'apprentissage.

73 C'est un travail minutieux, extrêmement précis qui, justement par ce fait, permet aux élèves de progresser à leur rythme, d'avancer petit à petit, sans être perdus face à la masse de ce qu'il faudrait faire, sans baisser les bras face à l'ampleur du désastre que constitue une copie farcie de stylo rouge, sans renoncer devant la maitrise univoque du professeur énonçant la correction-type. 

d'affiner sa lecture des textes, de mieux s'orienter dans les textes non narratifs, de passer à une analyse plus fine, ce qui ne manquera pas d'améliorer les retombées pédagogiques de son travail. Faire cette recherche sur des textes d'élèves, c'est enfin avoir accès de façon différente à un matériau considéré comme barbant, c'est ne plus tout le temps y lire les mêmes "bêtises", c'est s'approprier un matériau quantitativement important dans la vie du prof. A terme, c'est s'ennuyer moins et jouer sur la qualité de ce qu'on reçoit.

\section{RÉSUMÉS}

La correction apparaît comme une corvée à de nombreux enseignants. Le stage dont parle l'auteur tend à changer le regard de ceux-ci sur cet exercice en analysant, sous un jour nouveau, les démarches d'apprentissage mise en place par les élèves face à différents textes et par les professeurs face à la correction de copies. Il s'agit, notamment, pour les professeurs de comprendre que les consignes données peuvent être la cause de la mauvaise qualité des copies rendues. Brigitte Pons écrit, ici, à la fois en tant que stagiaire et en tant que formatrice.

\section{INDEX}

Mots-clés : évaluation, apprentissage, pédagogie, écrits scolaires

Keywords : education, rating, learning, school writings 